\title{
Music Streaming Services as Adjunct Therapies for Depression, Anxiety, and Bipolar Symptoms: Convergence of Digital Technologies, Mobile Apps, Emotions, and Global Mental Health
}

\author{
Karl Schriewer ${ }^{1}$ and Grzegorz Bulaj ${ }^{2 *}$ \\ 1 Juan Diego Catholic High School, Draper, UT, USA, ${ }^{2}$ Skaggs Pharmacy Institute, College of Pharmacy, University of Utah, \\ Salt Lake City, UT, USA
}

Keywords: wearable, medical device, telemedicine, antidepressant, randomized clinical trial, psychosocial, prevention, public health

\section{INTRODUCTION}

OPEN ACCESS

Edited by:

Joav Merrick,

Ministry of Social Affairs, Israel

Reviewed by:

Tammy Chung,

University of Pittsburgh Medical

Center, USA

Christophe Huynh,

Institut universitaire, Canada

Pier Luigi Lopalco,

University of Pisa, Italy

*Correspondence:

Grzegorz Bulaj

bulaj@pharm.utah.edu

Specialty section:

This article was submitted

to Digital Health,

a section of the journal

Frontiers in Public Health

Received: 01 July 2016 Accepted: 20 September 2016 Published: 30 September 2016

Citation:

Schriewer K and Bulaj G (2016) Music Streaming Services as Adjunct

Therapies for Depression, Anxiety, and Bipolar Symptoms: Convergence of Digital Technologies, Mobile Apps, Emotions, and Global Mental Health.

Front. Public Health 4:217.

doi: 10.3389/fpubh.2016.00217
Mobile technologies and music are recognized as opportunities to address mental health challenges (1-3), while clinical and economic benefits of mobile health (mHealth) are currently studied (4-6). Herein, we describe feasibility of repurposing music streaming services as therapies for affective disorders. According to the World Health Organization, there are 350 million people worldwide suffering from depression, and 60 million people living with bipolar disorder. Patients with affective disorders such as depression, anxiety, or bipolar spectrum, and their caregivers are challenged with managing disease symptoms, long-term treatments, and disabilities. Between 1990 and 2010, there has been a $41 \%$ increase in public health burden of mental, neurological, and substance use disorders, as measured by disability-adjusted life years (7). Depression accounts for $40.5 \%$ of total disabilityadjusted life years among mental and substance-use disorders, whereas anxiety and bipolar disorder account for 14.6 and 7\%, respectively (8). A long-term morbidity in bipolar spectrum disorders emphasizes the needs to improve treatments for depression (9). Treatments of affective disorders include mainly antidepressant, antipsychotic medications, and cognitive behavioral therapy (CBT). The efficacy of antidepressants for children and adolescent patients (10), medication adherence, and limited access to CBT in many countries continue to be a challenge for public health.

Treatments of depression, anxiety, and bipolar symptoms comprise CBT, psychosocial, and self-care interventions, also delivered via online and digital technologies $(7,11,12)$. Opportunities for developing mobile apps and web-based interventions for neurological and mental disorders are economically feasible and coincide with the global adoption rates for smartphones $(6,13-16)$. Promising findings from clinical testing of mobile apps, e.g., in depression (17), are accompanied by challenges in patient engagement (18) and alignment of clinical and digital contents (19). Converting a mobile phone into low-cost virtual reality devices (exemplified by a Google VR cardboard) extends its potential medical applications $(20,21)$. Growing number of health-related wearables and devices measuring electrodermal activity (EDA), heart rate variability, or mobile electroencephalogram (EEG) systems, expand the use of digital technologies in medicine, including mental health [a 5-week treatment with EEG-based musical neurofeedback improved depression scores by 17\% (22)]. Companies like Apple, Samsung, LG, Microsoft, Fitbit, Empatica, Emotiv, NeuroSky, or Muse develop smart watches and mobile EEG systems with health/wellness applications, whereas WellDoc, Akili Interactive, or Pear Therapeutics are engaged in converting mobile apps and games into medical device-based therapies for specific chronic diseases. Given accessibility of smartphones and the internet, we discuss opportunities for music streaming services to be developed as adjunct 
therapies and prevention of depressive, anxiety, and bipolar spectrum symptoms.

\section{EMOTIONS AND MUSIC STREAMING SERVICES}

Music modulates emotions by engaging several neurotransmitters and brain structures $(2,3,23)$, including the brain's reward and dopaminergic systems $(24,25)$. Music-induced emotions include happiness, relaxation, sadness, nostalgia, arousal, surprise, or irritation (Figure 1A). The relationships between musical structures and emotions are complex and can be described in terms of the BRECVEM mechanism (Brain stem reflexes, rhythmic entrainment, evaluative conditioning, contagion, visual imagery, episodic memory, and musical expectancy) (26). Music components such as tempo, dynamics, low/high pitches, satisfying rhythm, minor/ major key, or instrumentation can change listeners' arousal and valence, impacting her/his overall mood (Figure 1A). Musicinduced emotional arousal and pleasure can be enhanced by expectation and predictability (27). Musical anticipation, specific rhythmic units, tempo, lyrics, and voice can affect arousal (28-30). Noteworthy, the musical harmony can contribute to intercultural
A

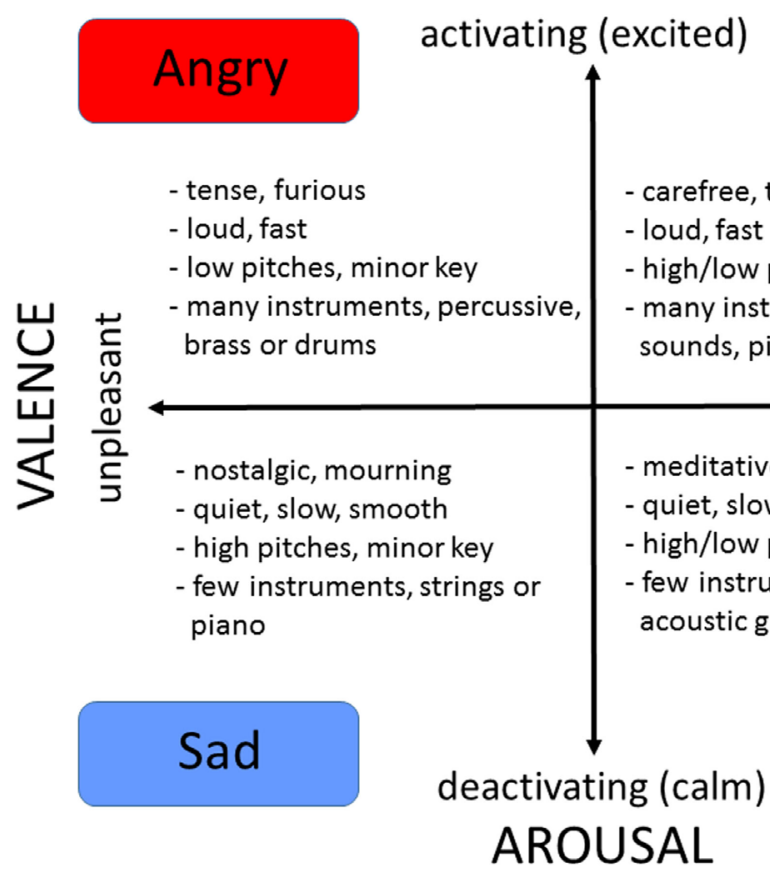

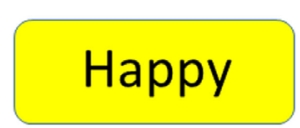

umphant

itches, major key

truments, electronic

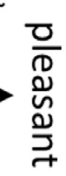

足 
differences in music perception (31). Specific musical structures such as those in the Mozart's Sonata K.448 were found to activate parasympathetic nervous system and to reduce the frequency of seizures and epileptiform discharges in epilepsy patients (32), and these antiseizure effects were observed in pediatric and adult patients across western and eastern cultures. The rhythm of the K.448 piece appeared to be one of key "active" structures, as determined by comparing it with the retrograde version of K.448 (33). Positive emotional effects of music were measured using plasma oxytocin and vasopressin levels, pointing to connections between the neuroendocrine system, music, and emotions (34). Music can modulate emotional circuitry in patients with major depressive disorder (35) and music listening for at least 3 weeks can reduce depressive symptoms, with some randomized controlled studies reporting $19-47 \%$ improvements in depression scores $(36,37)$.

Music streaming services such as Amazon Prime Music, Apple Music, Google Play Music, iHeartRadio, Pandora, Spotify, or SoundCloud play a variety of songs on-demand via the Internet and according to the user's interests and preferences. A person can select music stations based on specific songs, artists, genres, or mood of a song. The station then plays songs, which the listener already selected, as well as new songs, which are similar to previous songs played. Music streaming services offer millions of songs and musical tracks and are available worldwide, or in selected countries. Many stations offer mood-based categories, for example Spotify ("Have a Great Day," "Mood Booster," "Calm Down," "Good Vibes," etc.) and Apple Music ("Get Happy," "100 Most Uplifting Songs Ever") have preset playlists to choose, while Google Play Music ("Confident," "Calm," "Energetic," etc.), Getty Images ("Up/Positive," "Inspirational," etc.), and Aupeo ("Happy," "Dramatic," "Relaxing," etc.) have search criteria using mood to find specific music. Musicovery, another music streaming service, has a unique interactive interface similar to the arousal/valence plane (Figure 1A), in which the user can choose specifically what mood of music they would like. Music streaming services are compatible with Android and iOS operating platforms and include music video streaming like Vimeo or YouTube.

We hypothesize that digital and mobile technologies have advanced enough for repurposing music streaming service into a therapy for affective disorders by modulating arousal and valence via music-evoked emotions. While music streaming services have evolved to deliver on-demand music for entertainment purposes, many stations offer mood-based categories of music. It is unclear what criteria are used to choose specific pieces of music for particular mood-related categories, and they likely vary among music streaming services. Currently, there is a large diversity of mood-based stations, allowing a person to choose from, and listen to, a type of music that can affect her/his arousal (activating/deactivating) and valence (pleasant/unpleasant). As we describe below, before creating novel music libraries and streaming playlists for specific clinical purposes, individual pieces of music can be validated for their physiological responses using EDA and EEG systems.

Streaming music can modulate arousal and valence in people with affective disorders. For example, for patients with depression, who may otherwise prefer deactivating and negativevalence music (38), a long-term and daily stimulation with judiciously selected music (e.g., $>60-70 \%$ of activating/arousal and positive/valence content) may produce additional clinical benefits. Similarly, for patients with anxiety, a daily streaming of calming/relaxing music can stabilize stress hormone levels regulated by the hypothalamic-pituitary-adrenal axis $(2,23)$. For people with bipolar disorder during the euthymic stage, a daily 30-min streaming of balanced activating/deactivating music with positive valence may help sustaining homeostasis of emotions and prevent relapses. Figure 1B illustrates how music streaming services could become adjunct therapies for patients with depression, anxiety, and bipolar spectrum disorders.

\section{CONVERTING MUSIC STREAMING SERVICES AND MOBILE APPS INTO ADJUNCT THERAPIES}

Repurposing music streaming services into a medical treatment includes validation of clinical claims, followed by the regulatory clearance/approval for using software as a medical device (examples of the regulatory agencies include the European Medicines Agency or the Food and Drug Administration). In the United States, the Food and Drug Administration and the Federal Trade Commission ensure that marketing of digital health products is validated for specific conditions. Clinical validation of the musicgenerating software in randomized controlled trials is necessary to support the medical device status (Figure 1B). There are several mobile apps and games that have been cleared as medical device therapies for the patients with diabetes or stroke. To the best of our knowledge, there are no published data showing clinical efficacy of music streaming services for specific affective disorders.

To develop a music streaming service as an adjunct therapy for treatment of depression, anxiety, and bipolar spectrum, several parameters can be tested in randomized clinical trials, such as: (1) judicious selection of music with respect to arousal (activation/deactivation) and valence (pleasant/unpleasant), (2) total length of the therapy and daily duration of listening, and (3) proportion of arousal activating versus deactivating music delivered to patients daily and throughout the whole treatment. Such system is schematically illustrated in Figure 1B, and the key "medical" element is software with the approved medical device status. An affective brain-computer music interface provides an example of music-generating algorithms that could navigate affective trajectories for targeting desirable affective state during listening to music (39). Noteworthy, a mobile app delivering customized and downloaded music playlist for off-line use can be developed as mobile medical app. Coupling music with mobile EEG systems or EDA smartwatches offers additional means to increase the clinical efficacy of music-streaming by enhancing the physiologically active content through biofeedback mechanisms $(22,32,39,40)$.

Many patients with chronic medical conditions (diabetes, arthritis, neuropathic pain, schizophrenia, addiction, epilepsy, cardiovascular, cancer, and HIV/AIDS) who suffer from depression as comorbidity could also benefit from the music streaming therapies. Broader applications of music-evoked modulation of emotions via streaming include post-traumatic stress disorder, 
attention-deficit/hyperactivity disorder, autism, insomnia, neurorehabilitation, neurodegenerative, and developmental disorders. Since internet-based, self-help interventions may support prevention of depression (41), preventive medicine indications of music streaming may be also appealing for those at risk for affective disorders due to genetic, stress, adverse childhood experiences, and other environmental factors (42). Applications of music streaming can include postoperative pain management, given results from randomized trials on music interventions for analgesia in adult and pediatric patients $(43,44)$. When developing music-streaming medical software, protection of patient's electronic health records, cybersecurity, long-term patient engagement (18), as well as unhealthy and maladaptive effects of music shall be taken into account $(45,46)$.

Increasing awareness among artists, music, digital, health care, and pharma industries about medical applications of streaming music could spur cross-disciplinary efforts toward development of low-cost, add-on therapies for chronic medical conditions. Given technological advances, biofeedback-based screening of already-existing musical tracks can facilitate initial evaluation of their clinical utility $(47,48)$. Analogous to drug discovery efforts using high-throughput assays, or virtual screening, collections of music can be mined with EEG, or EDA, or computer-based algorithms to detect and categorize specific musical structures. From the economic perspective, significantly higher revenues of pharmaceutical, electronic, and healthcare industries, as compared to copyright/music industry, favor mutual benefits of collaborative efforts to integrate creative elements into medical treatments. Challenges in advancing music streaming as medical treatments include: (1) differences between a rapid pace of technological advancements in consumer electronic industries and constant creation of new songs and music, as compared to a slower pace of clinical research and regulatory processes, (2) differences in business cultures between music/electronic industries and healthcare (profit margins versus the patient safety and clinical efficacy), and (3) individual and cultural preferences for different genres of music. Since music streaming services offer millions of musical tracks, and they constantly update their playlist portfolios, the above challenges could be mitigated by: (1) developing clinically effective, music-selecting algorithms that are independent of technological, or music content, updates, and (2) long-term collaborations between pharma and music streaming companies. Clinical validation and regulatory approval of music streaming has an incentive of broader acceptance by the patients, health-care providers, including potential reimbursements by third-party payers. Integration of music and mobile

\section{REFERENCES}

1. Berrouiguet S, Baca-Garcia E, Brandt S, Walter M, Courtet P. Fundamentals for future mobile-health (mHealth): a systematic review of mobile phone and web-based text messaging in mental health. J Med Internet Res (2016) 18(6):e135. doi:10.2196/jmir.5066

2. Koelsch S. Brain correlates of music-evoked emotions. Nat Rev Neurosci (2014) 15(3):170-80. doi:10.1038/nrn3666

3. Koelsch S. Music-evoked emotions: principles, brain correlates, and implications for therapy. Ann N Y Acad Sci (2015) 1337:193-201. doi:10.1111/ nyas. 12684 software with pharmacological treatments can lead toward development of molecular-behavioral combination therapies via drug-device combination products $(14,49)$. Such strategy also expands future portfolio of therapies, in addition to repurposing existing drugs, or developing new drugs, which can cost between $\$ 2$ and $\$ 3$ billion (50). Music streaming has a potential to improve health-related quality of life of people living with chronic medical conditions, further supporting public health.

\section{CONCLUSION}

The diversity of cultural origins, music genres, and personal preferences does not impact universal values of music, while music therapies have been known for many years. Herein, we describe how a rapid growth in internet and mobile technologies, including worldwide accessibility of music streaming and smartphones can in part address increasing global mental health challenges. Repurposing music streaming services into the therapies for depression, anxiety, or bipolar spectrum will require cross-disciplinary collaborations and rigorous clinical validation of specific medical claims. Given convenience and low costs of delivering digital interventions, developing music streaming therapies could offer new opportunities for patients, their caregivers, health-care professionals, music industry, and artists worldwide.

\section{AUTHOR CONTRIBUTIONS}

GB conceived the project. KS analyzed and reviewed music streaming services and music structures shown in Figure 1. GB and KS reviewed literature, discussed the data, and wrote the manuscript.

\section{ACKNOWLEDGMENTS}

We thank the ALSAM Foundation for supporting KS research internship. We also thank Drs. Skye McKennon, Michael Cottle, and Jeremiah Jones for helpful comments on the manuscript. We acknowledge many relevant studies, technologies, and music streaming services that are not described here due to size limitations of this article, and we apologize to all those authors for not referencing their works.

\section{FUNDING}

This work was supported by research internship from the ALSAM Foundation.

4. Daniel H, Sulmasy LS, Health and Public Policy Committee of the American College of Physicians. Policy recommendations to guide the use of telemedicine in primary care settings: an American College of Physicians position paper. Ann Intern Med (2015) 163(10):787-9. doi:10.7326/ M15-0498

5. Bergmo TS. How to measure costs and benefits of ehealth interventions: an overview of methods and frameworks. J Med Internet Res (2015) 17(11):e254. doi:10.2196/jmir.4521

6. Ruby A, Marko-Holguin M, Fogel J, Van Voorhees BW. Economic analysis of an internet-based depression prevention intervention. J Ment Health Policy Econ (2013) 16(3):121-30. 
7. Patel V, Chisholm D, Parikh R, Charlson FJ, Degenhardt L, Dua T, et al. Addressing the burden of mental, neurological, and substance use disorders: key messages from disease control priorities, 3rd edition. Lancet (2016) 387(10028):1672-85. doi:10.1016/S0140-6736(15)00390-6

8. Whiteford HA, Degenhardt L, Rehm J, Baxter AJ, Ferrari AJ, Erskine HE, et al. Global burden of disease attributable to mental and substance use disorders: findings from the Global Burden of Disease Study 2010. Lancet (2013) 382(9904):1575-86. doi:10.1016/S0140-6736(13)61611-6

9. Forte A, Baldessarini RJ, Tondo L, Vazquez GH, Pompili M, Girardi P. Long-term morbidity in bipolar-I, bipolar-II, and unipolar major depressive disorders. J Affect Disord (2015) 178:71-8. doi:10.1016/j.jad.2015.02.011

10. Cipriani A, Zhou X, Del Giovane C, Hetrick SE, Qin B, Whittington C, et al. Comparative efficacy and tolerability of antidepressants for major depressive disorder in children and adolescents: a network meta-analysis. Lancet (2016) 388(10047):881-90. doi:10.1016/S0140-6736(16)30385-3

11. Todd NJ, Jones SH, Hart A, Lobban FA. A web-based self-management intervention for bipolar disorder 'living with bipolar': a feasibility randomised controlled trial. J Affect Disord (2014) 169:21-9. doi:10.1016/j.jad.2014.07.027

12. Hutton P, Taylor PJ. Cognitive behavioural therapy for psychosis prevention: a systematic review and meta-analysis. Psychol Med (2014) 44(3):449-68. doi:10.1017/S0033291713000354

13. Jones SP, Patel V, Saxena S, Radcliffe N, Ali Al-Marri S, Darzi A. How Google's 'ten things we know to be true' could guide the development of mental health mobile apps. Health Aff(2014) 33(9):1603-11. doi:10.1377/hlthaff.2014.0380

14. Bulaj G. Combining non-pharmacological treatments with pharmacotherapies for neurological disorders: a unique interface of the brain, drug-device, and intellectual property. Front Neurol (2014) 5:126. doi:10.3389/fneur.2014.00126

15. Anthes E. Mental health: there's an app for that. Nature (2016) 532(7597):20-3. doi:10.1038/532020a

16. Bakker D, Kazantzis N, Rickwood D, Rickard N. Mental health smartphone apps: review and evidence-based recommendations for future developments. JMIR Ment Health (2016) 3(1):e7. doi:10.2196/mental.4984

17. Birney AJ, Gunn R, Russell JK, Ary DV. MoodHacker mobile web app with email for adults to self-manage mild-to-moderate depression: randomized controlled trial. JMIR Mhealth Uhealth (2016) 4(1):e8. doi:10.2196/ mhealth.4231

18. Anguera JA, Jordan JT, Castaneda D, Gazzaley A, Arean PA. Conducting a fully mobile and randomised clinical trial for depression: access, engagement and expense. BMJ Innov (2016) 2(1):14-21. doi:10.1136/bmjinnov-2015-000098

19. Nicholas J, Larsen ME, Proudfoot J, Christensen H. Mobile apps for bipolar disorder: a systematic review of features and content quality. J Med Internet Res (2015) 17(8):e198. doi:10.2196/jmir.4581

20. Amado I, Brenugat-Herne L, Orriols E, Desombre C, Dos Santos M, Prost Z, et al. A serious game to improve cognitive functions in schizophrenia: a pilot study. Front Psychiatry (2016) 7:64. doi:10.3389/fpsyt.2016.00064

21. Sharar SR, Alamdari A, Hoffer C, Hoffman HG, Jensen MP, Patterson DR. Circumplex model of affect: a measure of pleasure and arousal during virtual reality distraction analgesia. Games Health J(2016) 5(3):197-202. doi:10.1089/ g4h.2015.0046

22. Ramirez R, Palencia-Lefler M, Giraldo S, Vamvakousis Z. Musical neurofeedback for treating depression in elderly people. Front Neurosci (2015) 9:354. doi:10.3389/fnins.2015.00354

23. Chanda ML, Levitin DJ. The neurochemistry of music. Trends Cogn Sci (2013) 17(4):179-93. doi:10.1016/j.tics.2013.02.007

24. Salimpoor VN, Benovoy M, Larcher K, Dagher A, Zatorre RJ. Anatomically distinct dopamine release during anticipation and experience of peak emotion to music. Nat Neurosci (2011) 14(2):257-62. doi:10.1038/nn.2726

25. Zatorre RJ, Salimpoor VN. From perception to pleasure: music and its neural substrates. Proc Natl Acad Sci U S A (2013) 110(Suppl 2):10430-7. doi:10.1073/ pnas. 1301228110

26. Juslin PN, Barradas G, Eerola T. From sound to significance: exploring the mechanisms underlying emotional reactions to music. Am J Psychol (2015) 128(3):281-304. doi:10.5406/amerjpsyc.128.3.0281

27. van den Bosch I, Salimpoor VN, Zatorre RJ. Familiarity mediates the relationship between emotional arousal and pleasure during music listening. Front Hum Neurosci (2013) 7:534. doi:10.3389/fnhum.2013.00534

28. Vuust P, Gebauer LK, Witek MA. Neural underpinnings of music: the polyrhythmic brain. Adv Exp Med Biol (2014) 829:339-56. doi:10.1007/ 978-1-4939-1782-2_18
29. Fernandez-Sotos A, Fernandez-Caballero A, Latorre JM. Influence of tempo and rhythmic unit in musical emotion regulation. Front Comput Neurosci (2016) 10:80. doi:10.3389/fncom.2016.00080

30. Loui P, Bachorik JP, Li HC, Schlaug G. Effects of voice on emotional arousal. Front Psychol (2013) 4:675. doi:10.3389/fpsyg.2013.00675

31. McDermott JH, Schultz AF, Undurraga EA, Godoy RA. Indifference to dissonance in native Amazonians reveals cultural variation in music perception. Nature (2016) 535(7613):547-50. doi:10.1038/nature18635

32. Lin LC, Lee WT, Wu HC, Tsai CL, Wei RC, Jong YJ, et al. Mozart K.448 and epileptiform discharges: effect of ratio of lower to higher harmonics. Epilepsy Res (2010) 89(2-3):238-45. doi:10.1016/j.eplepsyres.2010.01.007

33. Xing Y, Xia Y, Kendrick K, Liu X, Wang M, Wu D, et al. Mozart, mozart rhythm and retrograde mozart effects: evidences from behaviours and neurobiology bases. Sci Rep (2016) 6:18744. doi:10.1038/srep18744

34. Dai L, Carter CS, Ying J, Bellugi U, Pournajafi-Nazarloo H, Korenberg JR. Oxytocin and vasopressin are dysregulated in Williams syndrome, a genetic disorder affecting social behavior. PLoS One (2012) 7(6):e38513. doi:10.1371/ journal.pone.0038513

35. Lepping RJ, Atchley RA, Chrysikou E, Martin LE, Clair AA, Ingram RE, et al. Neural processing of emotional musical and nonmusical stimuli in depression. PLoS One (2016) 11(6):e0156859. doi:10.1371/journal.pone.0156859

36. Chan MF, Wong ZY, Onishi H, Thayala NV. Effects of music on depression in older people: a randomised controlled trial. J Clin Nurs (2012) 21(5-6):776-83. doi:10.1111/j.1365-2702.2011.03954.x

37. Chan MF, Wong ZY, Thayala NV. The effectiveness of music listening in reducing depressive symptoms in adults: a systematic review. Complement Ther Med (2011) 19(6):332-48. doi:10.1016/j.ctim.2011.08.003

38. Sachs ME, Damasio A, Habibi A. The pleasures of sad music: a systematic review. Front Hum Neurosci (2015) 9:404. doi:10.3389/fnhum.2015.00404

39. Daly I, Williams D, Kirke A, Weaver J, Malik A, Hwang F, et al. Affective brain-computer music interfacing. J Neural Eng (2016) 13(4):046022. doi:10.1088/1741-2560/13/4/046022

40. Greco A, Valenza G, Lanata A, Rota G, Scilingo EP. Electrodermal activity in bipolar patients during affective elicitation. IEEE J Biomed Health Inform (2014) 18(6):1865-73. doi:10.1109/JBHI.2014.2300940

41. Buntrock C, Ebert DD, Lehr D, Smit F, Riper H, Berking M, et al. Effect of a web-based guided self-help intervention for prevention of major depression in adults with subthreshold depression: a randomized clinical trial. JAMA (2016) 315(17):1854-63. doi:10.1001/jama.2016.4326

42. Chan RC, Kringelbach ML. Editorial: at risk for neuropsychiatric disorders: an affective neuroscience approach to understanding the spectrum. Front Behav Neurosci (2016) 10:165. doi:10.3389/fnbeh.2016.00165

43. van der Heijden MJ, Oliai Araghi S, van Dijk M, Jeekel J, Hunink MG. The effects of perioperative music interventions in pediatric surgery: a systematic review and meta-analysis of randomized controlled trials. PLoS One (2015) 10(8):e0133608. doi:10.1371/journal.pone.0133608

44. Dabu-Bondoc S, Vadivelu N, Benson J, Perret D, Kain ZN. Hemispheric synchronized sounds and perioperative analgesic requirements. Anesth Analg (2010) 110(1):208-10. doi:10.1213/ANE.0b013e3181bea424

45. Carlson E, Saarikallio S, Toiviainen P, Bogert B, Kliuchko M, Brattico E. Maladaptive and adaptive emotion regulation through music: a behavioral and neuroimaging study of males and females. Front Hum Neurosci (2015) 9:466. doi:10.3389/fnhum.2015.00466

46. Saarikallio S, Gold C, McFerran K. Development and validation of the HealthyUnhealthy Music Scale. Child Adolesc Ment Health (2015) 20(4):210-7. doi:10.1111/camh.12109

47. Mihajlovic V, Grundlehner B, Vullers R, Penders J. Wearable, wireless EEG solutions in daily life applications: what are we missing? IEEE J Biomed Health Inform (2015) 19(1):6-21. doi:10.1109/JBHI.2014.2328317

48. Dillon A, Kelly M, Robertson IH, Robertson DA. Smartphone applications utilizing biofeedback can aid stress reduction. Front Psychol (2016) 7:832. doi:10.3389/fpsyg.2016.00832

49. Bulaj G, Ahern MM, Kuhn A, Judkins ZS, Bowen RC, Chen Y. Incorporating natural products, pharmaceutical drugs, self-care and digital/mobile health technologies into molecular-behavioral combination therapies for chronic diseases. Curr Clin Pharmacol (2016) 11(2):128-45. doi:10.2174/157488471 1666160603012237

50. Nosengo N. Can you teach old drugs new tricks? Nature (2016) 534(7607): 314-6. doi:10.1038/534314a 
Conflict of Interest Statement: GB is a cofounder and the officer of Epicadence PBC, Public Benefit Corporation, a company developing music and mobile software as medical device therapy for epilepsy patients [this technology was previously described in Ref. (14)]. GB is also a co-inventor of patent-pending technologies "Disease Therapy Game Technology" and "Multimodal Epilepsy Management Suite." In patent-pending application "Multimodal Epilepsy Management Suite," there is a description of music streaming for epilepsy patients. KS declares no conflict of interest.
Copyright (c) 2016 Schriewer and Bulaj. This is an open-access article distributed under the terms of the Creative Commons Attribution License (CC BY). The use, distribution or reproduction in other forums is permitted, provided the original author(s) or licensor are credited and that the original publication in this journal is cited, in accordance with accepted academic practice. No use, distribution or reproduction is permitted which does not comply with these terms. 